\title{
BMJ Open Factors affecting the acceptability of isoniazid preventive therapy among healthcare providers in selected HIV clinics in Nairobi County, Kenya: a qualitative study
}

\author{
Elvis Omondi Achach Wambiya, ${ }^{1,2}$ Martin Atela, ${ }^{3,4}$ Ejemai Eboreime, ${ }^{2,5}$ \\ Latifat Ibisomi ${ }^{2,6}$
}

To cite: Wambiya EOA, Atela M, Eboreime $\mathrm{E}$, et al. Factors affecting the acceptability of isoniazid preventive therapy among healthcare providers in selected HIV clinics in Nairobi County, Kenya: a qualitative study. BMJ Open 2018;8:e024286. doi:10.1136/ bmjopen-2018-024286

- Prepublication history for this paper is available online. To view these files, please visit the journal online (http://dx.doi. org/10.1136/bmjopen-2018024286).

Received 18 May 2018 Revised 5 November 2018 Accepted 9 November 2018

D) Check for updates

(c) Author(s) (or their employer(s)) 2018. Re-use permitted under CC BY-NC. No commercial re-use. See rights and permissions. Published by BMJ.

For numbered affiliations see end of article.

Correspondence to Mr Elvis Omondi

Achach Wambiya; eowambiya@gmail.com

\section{ABSTRACT}

Objective Despite being globally recommended as an effective intervention in tuberculosis (TB) prevention among people living with HIV, isoniazid preventive therapy (IPT) implementation remains suboptimal, especially in sub-Saharan Africa. This study explored the factors influencing the acceptability of IPT among healthcare providers in selected HIV clinics in Nairobi County, Kenya, a high HIV/TB burden country.

Design A qualitative study was conducted using indepth interviews with healthcare providers in selected HIV clinics. All conversations were audio recorded, transcribed verbatim and analysed using a thematic approach.

Setting The study was conducted in the HIV clinics of three purposefully selected public healthcare facilities in Nairobi County, Kenya between February 2017 and April 2017.

Participants Eighteen purposefully selected healthcare providers (clinicians, nurses, pharmacists and counsellors) working in the HIV clinics participated in the study.

Results Provider acceptability of IPT was influenced by factors relating to the organisational context, provider training on IPT and their perception on its efficacy, length and clarity of IPT guidelines and standard operation procedures, as well as structural factors (policy, physical and work environment). Inadequate high-level commitment and support for the IPT programme by programme managers and policy-makers were found to be the major barriers to successful IPT implementation in our study context.

Conclusion This study provides insight into the complexity of factors affecting the IPT implementation in Kenya. Ensuring optimal acceptability of IPT among healthcare providers will require an expanded depth of engagement by policy-makers and IPT programme managers with both providers and patients, as well as on-the-job design specific actions to support providers in implementation. Such high-level commitment and support are consequently essential for quality delivery of the intervention.
Strengths and limitations of this study

- To our knowledge, this is among the first qualitative studies exploring factors influencing the acceptability of isoniazid preventive therapy among healthcare providers in the context of HIV clinics providing integrated HIV and tuberculosis services.

- The inclusion of both clinical and non-clinical healthcare providers in the study enabled the collection of information at different levels and cadres of health service provision thereby enhancing the breadth and validity of the information obtained.

- The adaptation of existing theory and literature to guide the study enabled the collection of context-specific information at different levels of the health system.

- Purposive selection of the health facilities included in the study may limit the generalisability of our findings beyond the study context. However, the conclusions and recommendations are useful and applicable in other contexts.

\section{INTRODUCTION}

Tuberculosis (TB) and HIV coinfection remain a major public health threat and challenge to health systems in many low-income and middle-income countries (LMICs). According to WHO, people living with HIV (PLHIV) accounted for about $10 \%$ of the 10.4 million reported TB cases in 2016. ${ }^{1}$ PLHIV are about 20-30 times more likely to develop active TB compared with those without HIV. Moreover, TB is the leading cause of death among PLHIV. In fact, 374000 deaths among PLHIV in 2016 were attributed to $\mathrm{TB} .{ }^{1} \mathrm{HIV}$ and $\mathrm{TB}$ coinfection also places an immense burden on health systems in LMICs and threatens global TB and HIV reduction targets. ${ }^{23}$ The HIV/TB coinfection burden is heaviest in sub-Sahara Africa. ${ }^{1}$ 
Kenya is one of the countries with high burden of TB, $\mathrm{HIV} / \mathrm{TB}$ and multidrug-resistant TB (MDR-TB). ${ }^{4}$ Overall TB incidence for Kenya was 169000 in 2016 and an incidence rate of 348 per 100000 population. ${ }^{15}$ Nonetheless, Kenya has made considerable progress in reducing the $\mathrm{HIV} / \mathrm{TB}$ coinfection rate which fell from $45 \%$ in 2008 to $30 \%$ in $2016 .{ }^{67}$ In 2015 , approximately $31 \%$ of persons who developed TB in Kenya were HIV infected. ${ }^{6}$

To reduce the burden of TB among PLHIV, WHO recommends three interventions collectively termed 'the Three I's for TB/HIV' namely: intensified TB case finding (ICF), isoniazid preventive therapy (IPT) and infection control for TB. ${ }^{89}$ IPT is an evidence-based intervention with proven effectiveness of reducing the risk of TB in PLHIV by $33 \%-62 \% .{ }^{10}$ It is recommended for individuals with documented latent infection with Mycobacterium tuberculosis to prevent its progression into an active disease, and for PLHIV in areas with high HIV prevalence and latent TB prevalence greater than $30 \%{ }^{8-10}$ IPT involves the provision of isoniazid (INH) tablets to PLHIV who are TB negative or have latent TB. The recommended dose is $10 \mathrm{mg} / \mathrm{kg}$ daily for children and up to $300 \mathrm{mg} /$ day for adults. ${ }^{112}$ WHO guidelines recommend at least 6 months of IPT for children and adults including pregnant women, PLHIV and those who have successfully completed TB treatment. ${ }^{13}$ In areas of high prevalence and transmission of TB among PLHIV, IPT is conditionally recommended for 36 months as a proxy for lifelong or continuous treatment. ${ }^{13}$

Kenya adopted the 6-month IPT regimen for eligible persons in 2012. ${ }^{14}$ However, IPT implementation for PLHIV started in 2012 at selected facilities under the US government supported initiative, the President's Emergency Plan For AIDS Relief. ${ }^{14}$ Countywide scale-up of IPT began in March 2015 with Siaya, Kisumu, Migori, Homa Bay and Nairobi being the pioneer counties due to the high HIV prevalence rates in these counties. ${ }^{6}$ The roll-out was accompanied by an ambitious countrywide target of enrolling 90\% of PLHIV on IPT by December 2016. ${ }^{6}$ Implementation is supported by various cadres of healthcare providers. IPT is prescribed by a registered clinician (usually clinical officers in most HIV clinics), who also assesses IPT eligibility by ruling out contraindications such as peripheral neuropathy or liver disease and recommend confirmatory laboratory tests if deemed necessary. Nurses are involved in measuring vital signs and linking new patients to care. Clinicians and nurses are also involved in ICF procedure using a standard Ministry of Health standard ICF/IPT screening tool. They also monitor the treatment of patients who remain in care and update their IPT registers. Counsellors are involved in counselling new patients, caregivers (in the case of child patients) and patients who remain in care on the benefits of IPT to enhance adherence. Pharmacists dispense the drugs to the patients at initiation as well as during monthly refill visits. Social workers and community health volunteers are involved in contact tracing and linking both HIV and missing TB cases to care.
Despite the country's move to scale up IPT, there is widespread evidence of suboptimal implementation. ${ }^{6815}$ The latest IPT coverage survey indicated that only $3.6 \%$ of adults and $10 \%$ children eligible were initiated into IPT in 2015. ${ }^{6}$ While suboptimal IPT implementation is well documented, little is known about contextual factors that influence its implementation. Moreover, limited information exists on popular perceptions regarding its acceptability and factors influencing its application among healthcare providers in Kenya. Yet, it is widely recognised that healthcare providers are the front-line people delivering healthcare interventions and their acceptability is key to successful implementation and effectiveness of healthcare interventions. ${ }^{16}{ }^{17}$ This study responded to this gap through an in-depth analysis of the factors influencing the acceptability of IPT among healthcare providers in selected HIV clinics in Nairobi County, Kenya. Assessing IPT acceptability among healthcare providers can help to better understand the barriers and facilitators of IPT delivery at health facilities and therefore guide TB preventive care. Acceptability is also an important outcomes measure used to assess the effectiveness of implementation and to provide insights into how this contributes to health outcomes. ${ }^{18} 19$

The study adopted Proctor $e t a l$ s definition of acceptability as 'the perception among implementation stakeholders that a given treatment, service, practice or innovation is agreeable, palatable or satisfactory'. ${ }^{19}$

\section{METHODS}

\section{Study design}

This was a qualitative descriptive study using semistructured, in-depth interviews. The design, data collection, analysis and reporting were conducted in accordance with the Standards for Reporting Qualitative Research (SRQR). ${ }^{20}$

\section{Study setting}

The study was conducted in three facilities in Nairobi City County- 1 of the 47 counties in Kenya-with a population of about 3138369 people between February and April $2017 .{ }^{21}$ Nairobi County was selected because it was one of the pioneer Counties for the national roll-out of IPT in 2015. The study adopted a cross-sectional approach. Three public healthcare facilities (for purposes of anonymity coded as facility A, facility B and facility C) were purposefully selected based on physical location, size and the high volumes of patients with HIV and TB accessing integrated treatment services. Data were gathered through in-depth interviews with staff working in the HIV clinics referred to as comprehensive care centres (CCCs). At the time of the study, Facility A had about 45 health personnel of different cadres supporting 10226 patients with HIV in its CCC. The facility's IPT uptake was $70 \%$ in the last quarter of 2016. An average of 1974 patients visited the clinic per month in the last quarter of 2016. Similarly, facility B had about 25 healthcare providers in the CCC, 


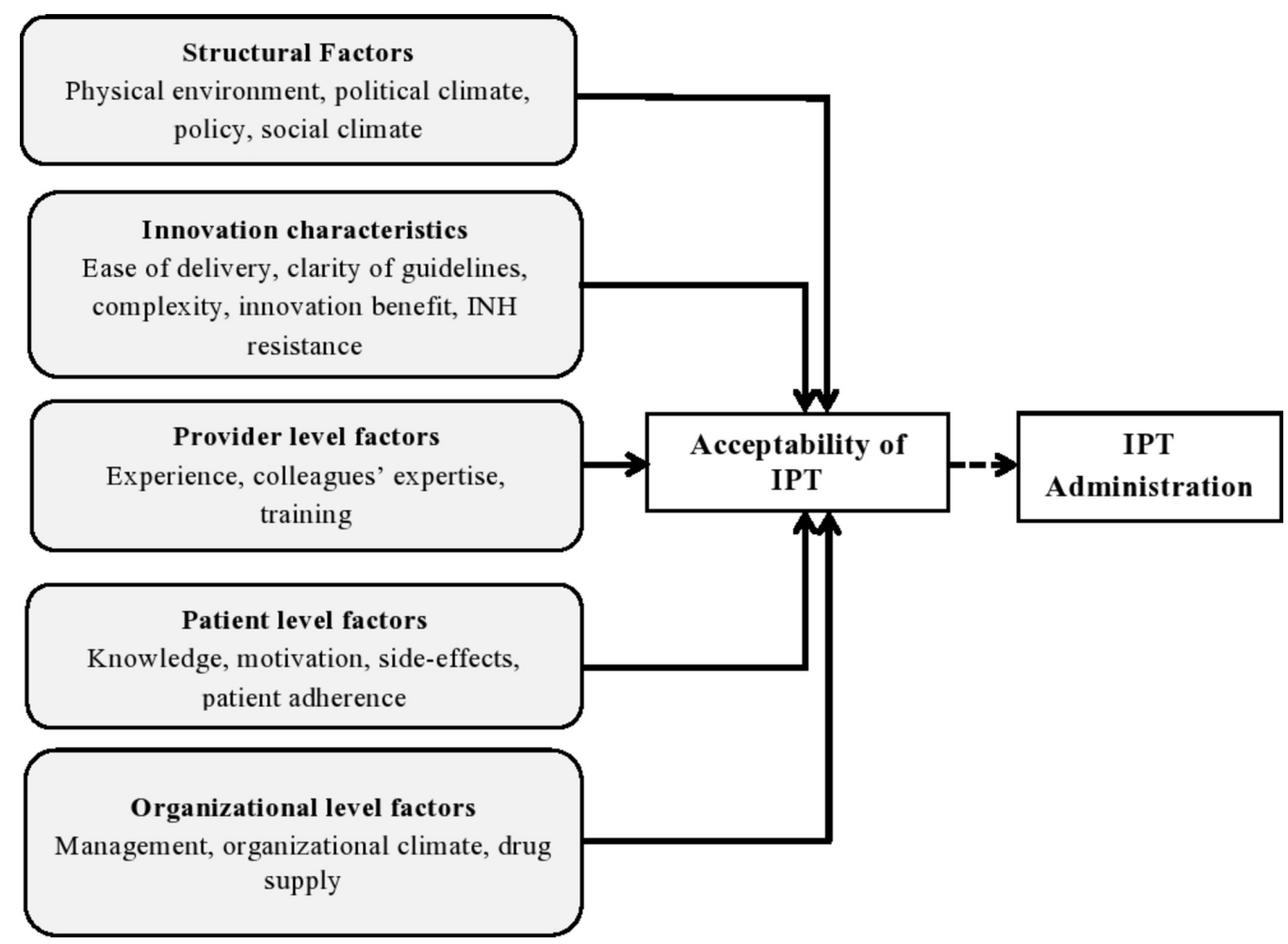

Figure 1 Conceptual framework of factors affecting the acceptability of IPT among healthcare providers. Adapted from Chaudoir et al. INH, isoniazid; IPT, isoniazid preventive therapy.

supporting 4860 patients and an IPT uptake of $68 \%$ in the last quarter of 2016. On the other hand, facility $\mathrm{C}$ had about 25 healthcare providers in the CCC, 1133 patients enrolled in care, $65 \%$ of whom were on IPT in the last quarter of 2016.

\section{Study participants}

The study involved 18 healthcare providers-14 clinicians (clinical officers, nurses and pharmacists) and 4 non-clinicians (counsellors) - working in the care centres of the selected health facilities. Respondents must have been involved in the IPT programme and worked in the clinic for at least 6 months prior to the study. Those who were absent during the study period were excluded. The providers were purposefully selected to ensure adequate representation in terms of gender, job cadre and length of stay at the facility. All consented to participate in the study.

\section{Sampling and recruitment}

Study participants were recruited through purposive sampling. This was facilitated by the lead researcher (EOAW) and the head nurses of the study HIV clinics. Prestudy meetings were convened in the clinics with facility managers and clinic staff to promote the study to eligible participants. Prospective participants were approached and contact established to agree on interview logistics such as dates, times and venues.

\section{Data collection}

In-depth interviews were conducted using an interview guide patterned after the themes of the conceptual framework of factors affecting the implementation outcomes by Chaudoir et al. ${ }^{22}$ The interviews were led by the lead researcher. The framework groups factors affecting the acceptability under five main categories: structural factors, innovation characteristics, provider-level factors, patient-level factors and organisational factors (figure 1). The interviews were privately conducted in the English language within the health facilities. Each session was approximately $45 \mathrm{~min}$ long and was audio recorded. Data were collected between February and April 2017.

\section{Research team and reflexivity}

The corresponding author (EOAW) is a data analyst, early career epidemiologist and implementation science researcher. EOAW was a graduate student and not affiliated to the sites at the time of the study. This provided confidence that the data obtained from the interviews were solely the participants' perceptions and not influenced by the previous contact. Other authors had no previous contact with the study sites. MA is a research scientist with interests in implementation science, health policy and systems strengthening research. EE is a health system, policy and implementation science researcher. LI is an associate professor in public health, with interests in demography and implementation science. All authors are well versed in mixed methods research approaches.

\section{Data analysis}

Audio-recorded transcripts were transcribed verbatim. Inductive thematic analysis was conducted. Data verification for accuracy and completeness was done through 
reading and rereading of the interview transcripts. Coding of the transcripts was done to identify themes, messages and patterns emerging from the data. The developed codes were matched to ensure integrity and similarity between the researchers. A codebook was developed after integration and collation of the identified codes. From the codebook, broader themes and subthemes that emerged from the data were identified and reviewed to ensure they were appropriate for the interpretation. ${ }^{23}$ As part of a validation process and to elicit feedback from the participants, an anonymised summary of the findings was shared with randomly selected participants.

A research permit was obtained from the National Commission for Science, Technology and Innovation to conduct the study in Nairobi County, Kenya. Participants were briefed about the study and their rights and provided with an information sheet. Permission to access the selected health facilities was obtained from the management of the respective health facilities.

\section{Patient and public involvement}

The study aimed to address factors affecting the acceptability of IPT among healthcare providers, an implementation outcome which may affect the delivery of the intervention to patients. The identified factors may help improve the quality of care for PLHIV by improving the implementation of IPT. Initial findings of the broader study were shared with healthcare providers. Findings of this study will be shared with a broader programme and scientific communities through dissemination workshops, conferences and summary fact sheets.

\section{RESULTS}

The demographic characteristics of the 18 healthcare providers who participated in the in-depth interviews are presented in table 1.
Factors affecting the acceptability of IPT among healthcare providers

Although healthcare providers considered IPT to be an important intervention in the provision of care for PLHIV, they indicated several concerns with IPT at different levels that challenged their comfort and satisfaction with the intervention. The factors are grouped and presented in the following categories: structural factors, innovation characteristics, provider, patient-related factors and organisational factors. These results are summarised in figure 2.

\section{Structural factors}

Structural factors relate to the wider policy environment as well as the physical and working environment of the healthcare providers.

\section{Inadequate high-level support for IPT implementation}

Most of the providers cited limited commitment at a policy level in ensuring effective implementation and streamlining of the IPT programme, which consequently demotivated providers. A majority of the providers stated that strong commitment and explicit support from the policy-makers and IPT programme managers was necessary for effective implementation of the programme. Areas of support identified included advocacy for IPT, improving the supply of INH drugs and proper monitoring and evaluation of the IPT.

...there is no initiative by those who are concerned in the TB programme. They need to make sure that they insist on IPT, and put some regulations or some rules to be followed to ensure IPT is given to every eligible patient... (Non-clinical health care provider) The people concerned should be more involved in the programme. We are giving IPT but they are not fully engaged. We don't get any feedback from them.

\begin{tabular}{|c|c|c|c|c|c|}
\hline Variable & Value & Facility A & Facility B & Facility C & Total \\
\hline Sex & Males & 5 & 1 & 1 & 7 \\
\hline \multirow[t]{3}{*}{ Job category } & Clinical officers & 3 & 3 & 2 & 8 \\
\hline & Nurses & 2 & 1 & 2 & 4 \\
\hline & Pharmacists & 1 & 1 & - & 2 \\
\hline \multirow{3}{*}{$\begin{array}{l}\text { Length of stay in } \\
\text { comprehensive care } \\
\text { centre (years) }\end{array}$} & $<1$ & - & - & 2 & 2 \\
\hline & $2-4$ & 1 & 1 & - & 2 \\
\hline & $>4$ & 7 & 5 & 2 & 14 \\
\hline Age (years) & $>50$ & 2 & - & 1 & 3 \\
\hline
\end{tabular}




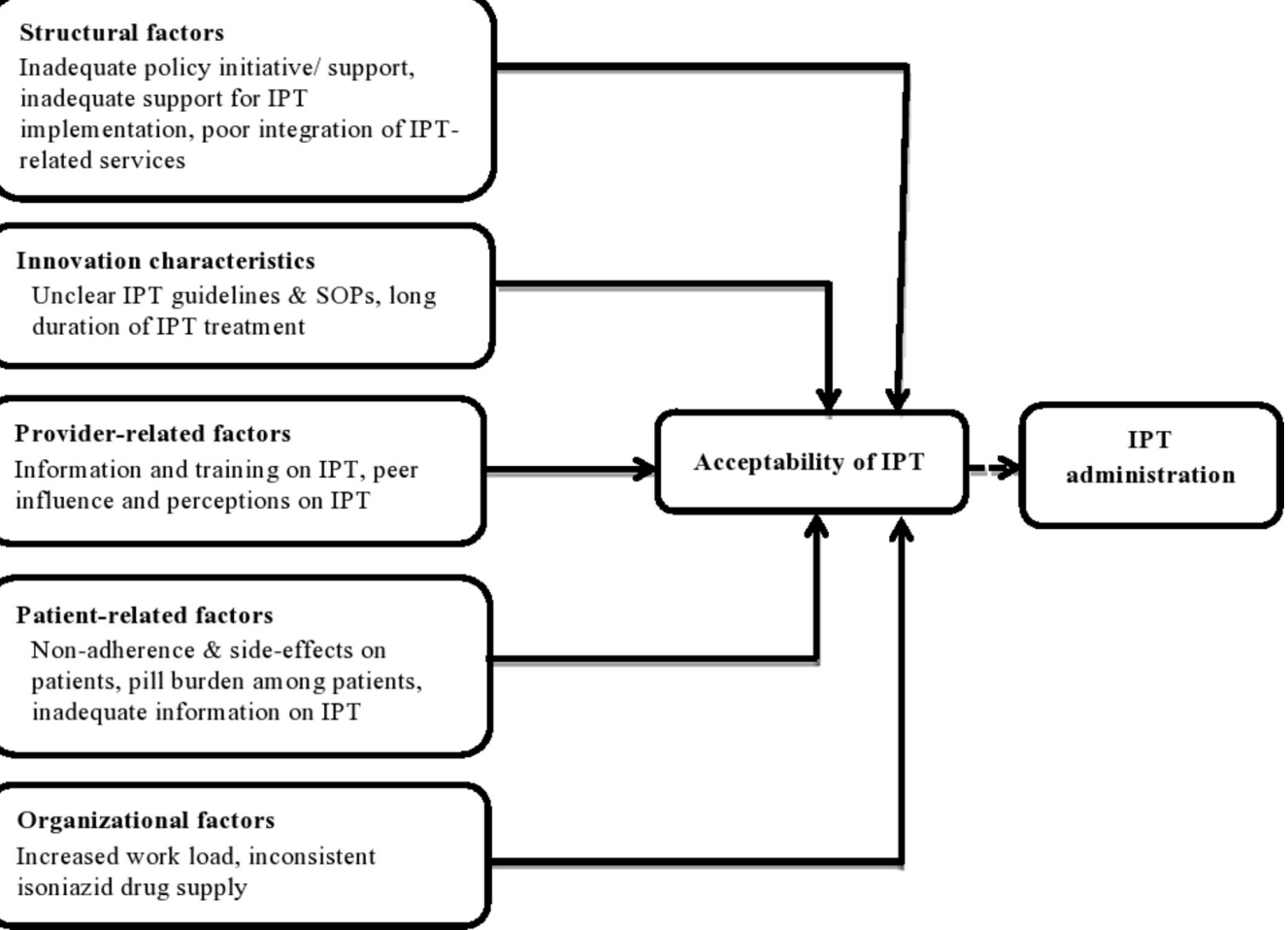

Figure 2 Conceptual framework of factors influencing the acceptability of IPT among healthcare providers in selected HIV clinics in Nairobi County, Kenya. IPT, isoniazid preventive therapy; SOPs, standard operating procedures.

They should monitor the supply of drugs and effects of IPT. (Clinical health care provider)

\section{Limited engagement with healthcare providers in the development} of IPT guideline

Providers lamented that there was pressure from policy-makers to implement the IPT policy guidelines during their introduction or revision at the CCCs without provider involvement. They noted that the consequence of this was to prescribe IPT without the full understanding its implications.

We were told these are the guidelines and we should follow...before they change the guidelines we should be involved...At the moment I don't feel like we are involved in this.... (Clinical health care provider)

\section{Poor integration of IPT-related services}

Most of the providers spoke of poor integration of IPT services in the clinic, noting that this hampered the delivery of the programme. It was noted that most of the clinical examinations required before IPT initiation were conducted in separate departments at additional costs. They felt that the examinations should be performed in the same facility and the costs subsidised to encourage uptake among patients. Most respondents felt that facility management and policy-makers had a key role in supporting the effective implementation of the programme.

....if we do [all] the tests from here, it will take like $30 \mathrm{~min}$ to do everything and give the patient IPT.
When they come again for check-ups, we can still do them again from here, and it takes less time and we get results in real time....it will even be faster for the patients (Clinical health care provider)

\section{Innovation characteristics}

Innovation factors relate to aspects of the intervention which enhance the chances of successful implementation. Discussions with healthcare providers revealed two main issues linked to IPT as an innovation that hampered its acceptance and implementation in their context. These are presented below.

\section{Unclear IPT guidelines and standard operating procedures (SOPS)}

Providers expressed discomfort with the IPT guidelines and SOPs citing lack of clarity. In particular, providers noted that guidelines on eligibility criteria, on how to decide whether a patient had active and latent TB and on the duration of IPT were unclear. Providers recommended a revision of the guidelines with specific regard to eligibility criteria and clarity on ruling out active and latent TB before prescription. There was also a lack of national consensus on IPT-related services as part of the $\mathrm{HIV} / \mathrm{TB}$ collaborative activities since some of the services differed among facilities. For instance, the provision of IPT with pyridoxine (to prevent peripheral neuropathy) versus IPT alone and monthly versus 3 monthly drug refills were reported to vary from facility to facility.

I think it [IPT] is a good idea but the problem is with the protocol, the SOPs. They are not very clear. 
They are not well documented...(Clinical health care provider)

...They told us in the training that we should give IPT every month to the patient. We are not comfortable with it...we prefer three month refill as we have been doing. Maybe they should re-evaluate these guidelines... (Clinical health care provider)

\section{Long duration of IPT}

Healthcare providers largely expressed discomfort with the long duration of the IPT treatment regimen. They reported this to be a critical factor that influenced their delivery of the intervention mainly because of pill burden and adverse effects reported by patients on long-term therapy. Most respondents recommended a reduced duration of the drug with the help of suitable research.

If I had a chance, I would give an IPT that would be taken once. Not the daily one for six months. That's a long time... (Clinical health care provider)

\section{Provider-related factors}

Factors related to individual healthcare providers such as experience and knowledge of IPT and peer influence also had considerable bearing on the perception and implementation of IPT in the clinics.

\section{Provider information and training on IPT}

Both clinical and non-clinical providers indicated that they needed to be empowered on the administration of IPT through additional information and training. Some providers cited limited or no specific training on IPT administration, which limited their ability to deliver the intervention. They recommended revision of guidelines and additional training on IPT, driven by policy-makers as well as regular monitoring and reporting of IPT outcomes from research to guide implementation.

...Some of us have not been taken through training on IPT. It was just introduced and you are told, 'Give IPT for this duration' ... I feel we should have been taken through training to know more about the IPT even before rolling it out. (Clinical health care provider)

I have never attended any training. It is just what I read in school and in books. We should be included in IPT training here. It would help a lot.(Non-clinical health care provider)

\section{Peer influence and perceptions on IPT}

The satisfaction of other healthcare providers with the intervention influenced their colleagues in the CCCs. Negative perceptions or doubts about the intervention by some healthcare providers affected the perception and delivery of IPT by the fellow providers.

...Colleagues say that patients tell them 'I've seen a friend of my husband who took [IPT] ..., you know. So that experience with my colleagues from the patients' mouth talking.... in fact, part of it was the reason why this facility delayed as a hospital to start IPT. (clinical health care provider)

\section{Patient-related factors}

Factors relating to the patients were thought to considerably affect healthcare providers' perceptions and delivery of IPT. The following are healthcare providers' reported patient-related factors affecting provider acceptability of IPT.

\section{Non-adherence to IPT and IPT side effects on patients}

Non-adherence to IPT after initiation by patients was considered a demotivating factor in administering IPT. Non-adherence was attributed to fear of side effects and pill burden among the patients. These views were shared by both the clinical and non-clinical providers. Providers also reported that some patients stopped using the therapy as a result of reported side effects. These discouraged other patients who became aware of these side effects from enrolling on the programme. Participants expressed concern that non-adherence would eventually lead to the development of resistance to INH drugs, in the long run, resulting in MDR-TB or extensively drug-resistant TB. Non-adherence was thought to be more likely among patients with a poor immunological, virological and clinical state as well as those on second-line antiretroviral therapy, which made healthcare providers reluctant in initiating IPT to these patients. Respondents recommended considerations of patient clinical state and drug regimen and argued for these to be added to the IPT guidelines.

...At least for them to do a research and find out if these side-effects are really associated with IPT. But if it is found to be safe to use, I would not have any other recommendations...Uptake reduced because they were not starting anyone else on IPT for fear of side-effects and death. (Clinical health care provider)

\section{Pill burden among patients}

Health care providers also felt that IPT increased the pill burden among the patients which affected patients' adherence to the medication. Providers described cases where patients complained of the difficulty in adhering to isoniazid drugs while some completely declined to take the medication due to the high number of pills prescribed for PLHIV. As a result, providers recommended that a formulation of IPT with shorter duration for the patients.

... patients feel that these drugs are so many and some say they don't want to start these drugs altogether... (Clinical health care provider)

...if they can review the concentration now, then maybe find out the concentration that can still work and still be mild to the patients...because of the pill burden to these clients... (Non-clinical health care 
provider)

Inadequate patient information on IPT

Information about the benefits and effects of IPT was reported to be limited among the patients. This resulted in rumours and misconceptions about IPT among the patients which led some patients to refuse to be initiated or to dispose of the medication even after being counselled. Providers expressed concern over the lack of consensus and support regarding patient education activities in the CCCs for IPT.

...We should conduct continuous medical education, and review how we provide patients with information ... (Non-clinical health care provider)

I think they need to do more education to the people... actually, most clients decline because they have never heard about it...they would say 'I am being treated for TB yet I don't have TB signs (Clinical health care provider)

\section{Organisational factors}

Organisational factors encompass factors related to the organisational context where IPT is implemented, in this case, the CCCs. These factors affect the effective implementation of IPT programmes.

\section{Increased workload}

Most clinical providers complained of the high workload in the facility, which they felt negatively affected implementation of the IPT programme. Providers reported that the limited number of clinicians did not match the high volume of patients in the CCC. The procedures to be conducted on the patient before IPT initiation were also considered very long and hence a burden to a single clinician. Providers called for the hiring of staff to be dedicated to IPT related activities in the CCCs.

...by the time you do all the screening for conditions like hepatitis, before even convincing the patient to start IPT... it is a big workload because we have many patients waiting in line to be served. (Clinical health care provider)

To comment about the environment and the working condition, here we have very heavy workload...then if you follow the standard operating procedures to give IPT, it will take you very long to complete all those investigations, examinations and what have you... (Clinical health care provider)

\section{Inconsistent INH drug supply}

Providers also mentioned stock-out of INH medication and other supplies related to the IPT programme in the facilities as a major impediment to effective implementation and acceptance of the therapy. They reported stockouts in the previous year and considered this a factor that greatly affected IPT delivery. Some providers felt that the erratic stocks and poor supply of the medications indicated lack of support for the IPT programme among policy-makers and management. This, in turn, negatively affected their perception, morale and delivery of the therapy.

...We started the programme nicely, empowering patients, counselling them on IPT, and encouraging them to take IPT... and then all of a sudden from nowhere, IPT drugs are not available (clinical health care provider)

My biggest challenge with the management [in CCC] is when there is erratic supply of IPT...So the patients were out of medication for some time and when you send them out to buy them; of course it's not possible for them to get the drug... (Clinical health care provider)

\section{DISCUSSION}

This study assessed the factors associated with provider acceptability of IPT in selected clinics in Nairobi City County, Kenya. Based on an adapted framework, identified factors have been grouped into five broad categories, viz. structural factors, innovation characteristics, provider, patient-related factors and organisational factors.

Among these findings, limited high-level commitment and support for the IPT programme by higher programme managers and policy-makers stood out as perhaps the biggest barrier to successful IPT implementation. Discussions with healthcare providers reaffirmed previous findings that supportive supervision, consistent a engagement between policy-makers and higher level supervisors with healthcare providers as well as the in-depth interaction between policy-makers and practitioners remain crucial for effective IPT implementation. The findings support previous evidence from similar contexts that showed that poor monitoring and lack of supervision of the IPT programme by higher managers influence IPT uptake. ${ }^{11} 24$

Linked to high-level commitment and support is provider involvement in the formulation of policies and guidelines. The study found limited involvement of the healthcare providers in the enactment and implementation of the IPT guidelines. As a result, most respondents were not comfortable implementing the guidelines in their clinics. Since only a few had received training and/ or support in IPT implementation, they saw it as a challenge rather than an opportunity to improve the health of their clients. In fact, providers across all the three facilities expressed their frustrations that they were being pressured to implement and deliver an intervention whose origin or implications they knew little about. The lukewarm ownership of IPT among providers as a result of the limited engagement at the design stage is not surprising and reinforces evidence in this area suggesting that successful implementation and compliance with such initiatives require mechanisms that help enforce official guidelines, address capacity gaps, and enhance provider and patient awareness. ${ }^{25}$ 
Another important finding from this study is how the nature of interventions (in this case IPT) affect implementation outcomes. The lack of clarity on some of the provisions of the guidelines meant that providers struggled to fully and effectively implement IPT provisions. This, in turn, negatively impacted their acceptability of the intervention. This finding resonates with evidence from other studies, ${ }^{24} 26$ and echoes the need for a wellplanned engagement process with caregivers whenever such guidelines are being developed and the need to make them as simple as possible.

Better integration of IPT-related services at the clinics could significantly improve the delivery of IPT. Integration could entail incorporation of all or most of the IPT-related procedures in one room/space. This can reduce challenges such as lost to follow-up in TB/HIV treatment thereby assisting healthcare providers to monitor the patients on IPT. Ultimately, this would lessen clinical provider workload. Lack of coordination between TB and HIV activities has been reported as a barrier to IPT implementation elsewhere. ${ }^{24}$ One study indicated that performing reading and interpreting tuberculin skin tests (TSTs) in the context of busy HIV clinics was a challenge for both patients and staff, negatively affecting the implementation of the IPT programme. ${ }^{27}$ In our study, providers questioned the efficacy of Kenya's IPT approach to identifying latent TB which involves a symptomatic algorithm using a standard Ministry of Health ICF/IPT screening tool and no TST for IPT eligibility. Healthcare providers have previously called for clarity of guidelines, showing that this is a major challenge to effective implementation and acceptance of IPT. ${ }^{27}$ This may also explain the lack of awareness among some providers of the benefits of IPT in some LMIC. ${ }^{24}$ Investigation of optimal duration, safety and efficacy of IPT and its role in reducing TB risk, particularly under programme conditions has been strongly recommended by WHO. ${ }^{28}$

Previous studies have hypothesised that provider-level factors could predict implementation outcomes. ${ }^{29}{ }^{30} \mathrm{In}$ our study, provider-related factors such as limited information and inadequate empowerment on IPT influenced the acceptability of IPT. A general lack of knowledge and experience with IPT have also been reported as primary barriers to IPT implementation in South Africa and Ethiopia. ${ }^{24}$ It is therefore important that provider training and information is prioritised for both clinical and non-clinical providers before implementation to achieve the desired outcomes. ${ }^{31}$ Providers in our study also reported that the lack of on-the-job training and support through mentorship and supportive supervision left them feeling inadequately equipped to handle emerging challenges associated with IPT implementation. These challenges highlight the need for tailor-made technical assistance during implementation including mentorship, retraining of the providers, training new staff, emotional support and mechanisms that take into consideration the contextual challenges.

Another important determinant of IPT acceptability among providers in the study location were patient-level predictors. In our context, poor adherence and pill burden among patients were key barriers to IPT acceptance among providers and patients (as reported by providers). Previous studies have associated poor adherence to IPT with INH resistance, which has made healthcare providers less likely to prescribe IPT. ${ }^{11}{ }^{32}$ Pill burden has also been perceived by healthcare providers as a cause of non-adherence causing them to be hesitant in prescribing IPT to patients with a high number of pills. ${ }^{1133}$ Adherence to IPT treatment is a critical factor to be considered when scaling treatment services, especially in areas with high TB incidence rates. Despite poor patient adherence being a key factor affecting acceptability, there was a lack of information among providers on evidence-based methods to monitor IPT adherence among patients. This might signify poor or lack of implementation of methods such as the use of treatment buddies, lay health providers, community-based directly observed preventive therapy to monitor and enhance IPT adherence. The availability of resources for close monitoring, supervision and evaluation of IPT outcomes is strongly recommended by WHO. ${ }^{34}$

Even though we used an exploratory approach to investigate IPT acceptance among providers, the providers reporting fear of INH drug resistance among patients should be cause for concern. This is particularly so because of the gradual increase in drug-resistant TB cases in Kenya (from 112 to 1300 in 2016). ${ }^{15}$ Policy-makers, healthcare providers and practitioners have questioned the implications of poor IPT adherence to drug-resistant TB disease especially in the case of long course INH monotherapy. ${ }^{35} 36$ To improve information on IPT among patients and boost uptake, a national advocacy and patient awareness is needed, among other interventions.

In the organisational context, the heavy workload on healthcare providers and INH stock-outs in the HIV clinics discouraged providers from initiating IPT, fearing poor adherence and associated side effects among their patients. Heavy workload among providers can often result in compromised quality and should be addressed as part of organisational context reforms to support IPT. In our study, this could be explained by the fact that the study clinics served a large population catchment area and not necessarily because the quality offered attracted patients to the clinics. Another reason for the heavy workload was inadequate staffing especially with regard to IPT trained staff. Ultimately, both factors affected the quality of care patients received.

The findings of this study have important policy implications. First, the lack of clarity of IPT guidelines highlights a need for revision and standardisation which would promote consensus among healthcare providers. Second, the findings highlight the need for strengthened monitoring and evaluation with a well-defined feedback mechanism of reporting by healthcare providers on IPT indicators. Finally, building both technical and logistic capacity in HIV clinics is important to improving the acceptability and ultimately the delivery of IPT. 
We recommend a number of interventions to improve healthcare provider acceptability in the study clinics and which may be explored in other similar contexts. First, involving healthcare providers in IPT guideline development and revision will make them more comfortable with implementation. Second, better integration of all IPT-related services in the same facility may help improve patient initiation, retention and follow-up of IPT. Additionally, training and continuous mentorship on IPT implementation for both clinical and non-clinical providers should be promoted in the health facilities to improve IPT acceptability and delivery.

\section{Strengths and limitations}

This study, to our knowledge, is among the first to assess the factors influencing the acceptability of IPT among healthcare providers in selected HIV clinics in Nairobi County, Kenya. The adaptation of existing theory and literature to guide the study enabled the collection of exhaustive context-specific information at different levels of the health system. The inclusion of both clinical and non-clinical personnel as key informants in the interviews enabled the collection of information at different levels and cadres of health service provision thereby enhancing the validity of the data. This study adhered to the SRQR.

Purposive selection of the health facilities may limit the generalisability of the findings from this study to other HIV clinics in Nairobi County. However, the study was context specific and the aim was to elicit in-depth information on IPT acceptability in this context, which may inform health service provision and policy in health systems of similar context.

Finally, the study was conducted among city hospitals, which are presumably better resourced as compared with those in other locations. Therefore, the IPT programme was expected to be better managed as opposed to other non-city HIV clinics. This could contribute to better acceptability of IPT among the providers whose concerns may not entirely reflect that of healthcare providers in other clinics in Nairobi County. Further studies aiming for generalisability should control for the tier of health facilities in assessing IPT acceptability.

\section{CONCLUSION}

The study gives an insight of the complexity of factors affecting IPT implementation and the value of qualitative methods and guiding frameworks to elucidate these factors. The acceptability of IPT among healthcare providers in this context was influenced by factors at different levels namely: structural factors, innovation characteristics, provider, patient-related factors and organisational factors. Ensuring optimal acceptability of IPT among healthcare providers will require a robust engagement with both providers and patients by policy-makers and IPT programme managers, as well as on-the-job design specific actions to support providers in implementation. This high-level commitment and support for IPT could improve provider acceptability and ultimately delivery of the intervention.

\section{Author affiliations}

${ }^{1}$ Research unit, African Population and Health Research Center, Nairobi, Kenya ${ }^{2}$ School of Public Health, University of the Witwatersrand, Johannesburg, South Africa

${ }^{3}$ Research Uptake \& Policy Engagement Unit, Partnership for African Social \& Governance Research, Nairobi, Kenya

${ }^{4}$ Public Health department, College of Health Sciences, University of Nairobi, Nairobi, Kenya

${ }^{5}$ Department of Planning Research \& Statistics, National Primary Health Care Development Agency, Abuja, Nigeria

${ }^{6}$ Research unit, Nigerian Institute of Medical Research (NIMR), Lagos, Nigeria

Acknowledgements The authors are grateful to the management and staff at comprehensive care centres of the selected health facilities for their cooperation, perspectives and support during the study. Much gratitude also goes to the School of Public Health, Faculty of Health Sciences at the University of the Witwatersrand, Johannesburg, South Africa for the immense support that facilitated the timely completion of the study.

Contributors EOAW, MA and LI contributed to the conceptualisation and design of the study including the development of the study tools. EOAW collected the data and did initial analysis and drafts of the manuscript. EE, MA and LI contributed to the data analysis, manuscript writing and editing. All authors read and approved the final version of the manuscript.

Funding This work was funded by TDR, the Special Programme for Research and Training in Tropical Diseases, which is hosted at WHO and cosponsored by UNICEF, UNDP, the World Bank and WHO. TDR grant number: B40299. First author ORCID ID: 0000-0002-4149-3417.

Competing interests None declared.

Patient consent for publication Not required.

Ethics approval Study approval and ethical clearance was obtained from the University of the Witwatersrand Human Research Ethics Committee (HREC) (approval No. M161164), Kenyatta National Hospital—University of Nairobi Ethics and Research Committee (approval No. P11/01/2017) and the Kenya Medical Research Institute Ethics and Research Committee (approval No. RES/7/3/1).

Provenance and peer review Not commissioned; externally peer reviewed. Data sharing statement No additional data are available.

Open access This is an open access article distributed in accordance with the Creative Commons Attribution Non Commercial (CC BY-NC 4.0) license, which permits others to distribute, remix, adapt, build upon this work non-commercially, and license their derivative works on different terms, provided the original work is properly cited, appropriate credit is given, any changes made indicated, and the use is non-commercial. See: http://creativecommons.org/licenses/by-nc/4.0/.

\section{REFERENCES}

1. World Health Organization. Global tuberculosis report 2017. Geneva: World Health Organization, 2017.

2. Sharma SK, Mohan A, Kadhiravan T. HIV-TB co-infection: epidemiology, diagnosis \& management. Indian J Med Res 2005;121:550-67.

3. Pawlowski A, Jansson M, Sköld M, et al. Tuberculosis and HIV coinfection. PLoS Pathog 2012;8:e1002464.

4. World Health Organization. Use of high burden country lists for TB by WHO in the post-2015 era. Geneva: World Health Organization, 2015.

5. Ministry of Health. Kenya tuberculosis prevalence survey. Kenya: Ministry of Health, 2016.

6. National Tuberculosis Leprosy and Lung Disease Program (NTLD-P). Annual report 2015. Kenya: National Tuberculosis Leprosy and Lung Disease Program (NTLD-P), 2015.

7. National AIDS and STI Control Program. Kenya HIV county profiles 2016. Kenya: National AIDS and STI Control Program, 2016.

8. World Health Organization. WHO policy on collaborative TB/HIV activities: guidelines for national programmes and other stakeholders. WHO policy on collaborative TB/HIV activities: guidelines for national programmes and other stakeholders, 2012. 
9. Getahun H, Van Gorkom J, Harries A, et al. Interim policy on collaborative TB/HIV activities, 2004.

10. World Health Organization. WHO THREE I's MEETING: Intensified Case Finding (ICF), Isoniazid Preventive Therapy (IPT) and TB Infection Control (IC) for people living with HIV. Geneva: World Health Organization, 2008.

11. Teklay G, Teklu T, Legesse B, et al. Barriers in the implementation of isoniazid preventive therapy for people living with HIV in Northern Ethiopia: a mixed quantitative and qualitative study. BMC Public Health 2016;16:840.

12. World Health Organization. Recommendation on 36 months isoniazid preventive therapy to adults and adolescents living with HIV in resource-constrained and high TB-and HIV-prevalence settings-2015 update, 2015.

13. World Health Organization. Guidelines for intensified tuberculosis case-finding and isoniazid preventive therapy for people living with HIV in resource-constrained settings. Geneva, 2011.

14. National Tuberculosis Leprosy and Lung Disease Program (NTLD-P). Isoniazid preventive therapy Kenya. 2016.

15. World Health Organization. Global tuberculosis report 2016. Geneva, Switzerland: World Health Organization, 2016.

16. Sekhon M, Cartwright M, Francis JJ. Acceptability of healthcare interventions: an overview of reviews and development of a theoretical framework. BMC Health Serv Res 2017;17:88.

17. Proctor EK, Landsverk J, Aarons G, et al. Implementation research in mental health services: an emerging science with conceptual, methodological, and training challenges. Adm Policy Ment Health 2009;36:24-34

18. Peters $\mathrm{DH}$, Adam $\mathrm{T}$, Alonge $\mathrm{O}$, et al. Implementation research: what it is and how to do it. BMJ 2013;347:f6753.

19. Proctor E, Silmere H, Raghavan R, et al. Outcomes for implementation research: conceptual distinctions, measurement challenges, and research agenda. Adm Policy Ment Health 2011;38:65-76.

20. O'Brien BC, Harris IB, Beckman TJ, et al. Standards for reporting qualitative research: a synthesis of recommendations. Acad Med 2014;89:1245-51.

21. Kenya National Bureau of Statistics. The 2009 Kenya population and housing census: population distribution by age, sex and administrative units. Kenya: Kenya National Bureau of Statistics, 2010.

22. Chaudoir SR, Dugan AG, Barr CH. Measuring factors affecting implementation of health innovations: a systematic review of structural, organizational, provider, patient, and innovation level measures. Implement Sci 2013;8:22.

23. Braun V, Clarke V. Using thematic analysis in psychology. Qual Res Psychol 2006;3:77-101.
24. Lester R, Hamilton R, Charalambous S, et al. Barriers to implementation of isoniazid preventive therapy in HIV clinics: a qualitative study. AIDS 2010;24(Suppl 5):S45-S48.

25. Atela $\mathrm{M}$, Bakibinga $\mathrm{P}$, Ettarh $\mathrm{R}$, et al. Strengthening health system governance using health facility service charters: a mixed methods assessment of community experiences and perceptions in a district in Kenya. BMC Health Serv Res 2015;15:539.

26. Makoni A, Chemhuru M, Tshimanga M, et al. Evaluation of the isoniazid preventive therapy (IPT) program in Shurugwi District, Midlands Province, Zimbabwe, January 2013 to August 2014. BMC Res Notes 2015;8:476.

27. Aït-Khaled N, Alarcon E, Bissell K, et al. Isoniazid preventive therapy for people living with HIV: public health challenges and implementation issues. Int J Tuberc Lung Dis 2009;13:927-35.

28. World Health Organization. The global plan to stop TB 2011 - 2015 Geneva: World Health Organization, 2011.

29. Durlak JA, DuPre EP. Implementation matters: a review of research on the influence of implementation on program outcomes and the factors affecting implementation. Am J Community Psychol 2008;41(3-4):327-50.

30. Damschroder LJ, Aron DC, Keith RE, et al. Fostering implementation of health services research findings into practice: a consolidated framework for advancing implementation science. Implement Sci 2009;4:50.

31. Wandersman A, Duffy J, Flaspohler P, et al. Bridging the gap between prevention research and practice: the interactive systems framework for dissemination and implementation. Am J Community Psychol 2008;41(3-4):171-81.

32. Mindachew M, Deribew A, Memiah $P$, et al. Perceived barriers to the implementation of Isoniazid preventive therapy for people living with HIV in resource constrained settings: a qualitative study. Pan Afr Med J 2014;17.

33. Makanjuola T, Taddese HB, Booth A. Factors associated with adherence to treatment with isoniazid for the prevention of tuberculosis amongst people living with HIV/AIDS: a systematic review of qualitative data. PLoS One 2014;9:e87166.

34. World Health Organization. Guide to monitoring and evaluation for collaborative TB/HIV Activities-2015 Update, 2015.

35. Date AA, Vitoria M, Granich R, Banda M, et al. Implementation of cotrimoxazole prophylaxis and isoniazid preventive therapy for people living with HIV. Bull World Health Organ 2010;88:253-9.

36. Woldehanna S, Volmink J. Treatment of latent tuberculosis infection in HIV infected persons. The Cochrane database of systematic reviews 2004;1:Cd000171. 